University of South Florida

DIGITAL COMMONS

Digital Commons @ University of

@ UNIVERSITY OF SOUTH FLORIDA

South Florida

1994

\title{
Comparison of TOPEX Sea Surface Heights and Tide Gauge Sea Levels
}

Gary T. Mitchum

University of Hawaii at Manoa, mitchum@usf.edu

Follow this and additional works at: https://digitalcommons.usf.edu/msc_facpub

Part of the Life Sciences Commons

\section{Scholar Commons Citation}

Mitchum, Gary T., "Comparison of TOPEX Sea Surface Heights and Tide Gauge Sea Levels" (1994). Marine Science Faculty Publications. 2080.

https://digitalcommons.usf.edu/msc_facpub/2080

This Article is brought to you for free and open access by the College of Marine Science at Digital Commons @ University of South Florida. It has been accepted for inclusion in Marine Science Faculty Publications by an authorized administrator of Digital Commons @ University of South Florida. For more information, please contact digitalcommons@usf.edu. 


\title{
Comparison of TOPEX sea surface heights and tide gauge sea levels
}

\author{
Gary T. Mitchum
}

Department of Oceanography, School of Ocean and Earth Science and Technology and Joint Institute for Marine and Atmospheric Research, University of Hawaii at Manoa

\begin{abstract}
TOPEX sea surface height data from the first 300 days of the mission are compared to sea level data from 71 tide gauges. The initial comparison uses sea surface height data processed according to standard procedures as defined in the users handbook. It is found that the median correlations for island and for coastal tide gauges are 0.53 and 0.42 , respectively. The analogous RMS differences between the two data sets are 7.9 and $10.4 \mathrm{~cm}$. The comparisons improve significantly when a 60-day harmonic is fit to the differences and removed. This period captures aliased $\mathbf{M}_{2}$ and $\mathbf{S}_{2}$ tidal energy that is not removed by the tide model. Making this correction and smoothing the sea surface height data over $25-\mathrm{km}$ along-track segments results in median correlations of 0.58 and 0.46 for the islands and coastal stations, and median RMS differences of 5.8 and $7.7 \mathrm{~cm}$, respectively. Removing once per revolution signals from the sea surface heights results in degraded comparisons with the sea levels. It is also found that a number of stations have poor comparisons due to propagating signals that introduce temporal lags between the altimeter and tide gauge time series. A final comparison is made by eliminating stations where this propagation effect is large, discarding two stations that are suspected to have problems with the sea level data, smoothing over 10-day intervals, and restricting attention to island gauges. This results in a set of 552 data pairs that have a correlation of 0.66 and a RMS difference of 4.3 $\mathrm{cm}$. The conclusion is that on timescales longer than about 10 days the RMS sea surface height errors are less than or of the order of several centimeters.
\end{abstract}

\section{Introduction}

The TOPEX altimeter provides measurements of sea surface height by differencing the satellite's range to the sea surface and the computed height of the satellite's orbit above a reference ellipsoid. While in principle the dynamically important sea surface topography can be obtained by correcting for the height of the ocean geoid relative to the reference ellipsoid, in practice inadequate knowledge of the geoid has led to the use of collinear and crossover analyses that provide only the temporal variations of the sea surface height. Thus at any point in the ocean that is overflown by the altimeter, a height time series with an arbitrary vertical reference point is obtained.

At the boundaries between land and sea, whether along the continental margins or at open ocean islands, the sea surface height variability can be measured by monitoring the height of the sea surface relative to a fixed point on the adjacent land. This is precisely what a tide gauge does, and these measurements are commonly referred to as sea level. The vertical reference point for sea level measurements is arbitrary and further requires an assumption that the land is not moving vertically. This latter assumption is not strictly true but is not a problem for the timescales (less than 1 year) to be discussed in this paper. Thus the sea level are very direct measurements of the sea surface height field at specific points and are the obvious source of independent data for

Copyright 1994 by the American Geophysical Union.

Paper number 94JC01640.

0148-0227/94/94JC-01640\$05.00 use in evaluating the altimetric heights. This paper describes an initial comparison of the TOPEX altimeter sea surface heights to the tide gauge sea levels.

It is interesting to note that before tide gauges became common in the latter half of the 19th century, sea level measurements were made by simply observing the height of the sea on a calibrated pole, which is often called a tide staff. Tide staff measurements are still taken at most tide gauge stations. Although these measurements are noisy, they are an admirably direct measurement of the sea level and can be considered the more fundamental measurements. This is because the staff measurements are used to monitor the vertical stability of the mechanical gauges, which are not assumed to be free of drifts of any sort, and to provide corrections if necessary. The tide gauge can thus be viewed as providing a temporal interpolation between the staff measurements. If altimetric heights are continuously compared to tide gauge data and found (or forced) to agree, one could argue that we are continuing this process by providing spatial interpolation to complement the temporal interpolation from the tide gauges.

Comparisons of the altimetric sea surface heights from Geosat to island sea levels have been done by many investigators [e.g., Tai et al., 1989; Cheney et al., 1989; Wyrtki and Mitchum, 1990; Harangozo et al., 1993; Chao et al., 1993]. A review of many of these comparisons with an emphasis on the tropical portions of the world's oceans has been provided by Mitchum and Kilonsky [1994]. The basic result of the various intercomparisons is that the Geosat sea surface heights correlate well with the sea level on times- 
cales longer than a few months and have a root-mean-square (RMS) difference of the order of $4-5 \mathrm{~cm}$.

It is important to note that these results were obtained only after applying various corrections for the errors in the computed orbits, and correcting for orbit errors was the focus of much attention for researchers using Geosat heights. For TOPEX this should not be true and other sources of error must be considered more carefully, as discussed by Koblinsky et al. [1992]. The tide model is an area of particular concern because the amplitude of the tides in the ocean are larger than most of the signals of interest. The Cartwright and Ray [1990] tide model provided on the TOPEX data tapes was developed from Geosat data and is the model used in all of the following analyses. Of course, the tide model is not the only source of error. For example, systematic differences were found between the Geosat heights and the sea level in the tropical Pacific that were consistent with poor corrections for water vapor changes in the tropical Pacific. This observation led to the suggestion that the altimetric heights and the sea level should be used in a "blended" fashion [Wyrtki and Mitchum, 1990]; that is, methods should be developed to use the tide gauges as tide staffs for the sea surface height measurements. I am not as concerned about this source of error for TOPEX because of the improved water vapor corrections available from the on-board radiometer.

There is another reason for carefully comparing sea surface heights from altimeters to sea level. By doing these comparisons it should be possible to identify tide gauges that do not properly represent open ocean height variability. Because tide gauges provide longer time series than altimeters, knowledge of which gauges could safely be taken as representative of large-scale sea surface height variability would be extremely valuable. I am inclined to be cautious in this respect, however, because the gauges are the more direct form of measurement. In the analyses in this paper a very conservative attitude is taken to eliminating gauges.

The approach taken in this paper is intended to result in estimates of the data quality that could be obtained by any scientist using the TOPEX data tapes and the accompanying handbook. What this means is that the initial altimeter data processing has followed the standard procedures recommended in the handbook rather than modifying the basic processing in order to optimize the comparisons. This philosophy is adopted in order to provide useful error estimates to the widest possible range of users. There are two primary objectives for this study. The first is to quantify the differences between the sea surface height and sea level data sets. The second objective is to understand the reasons for any mismatch between the two data sets. Since it is not possible to decide a priori which sea level records are expected to agree with open ocean sea surface height variability, an iterative approach is taken. A comparison is done using all gauges and the basic altimetric data. This comparison is refined by introducing additional steps into the altimetric processing. Finally, the comparison of the sea surface height to the sea level is done using only the gauges that are judged best suited to the task.

The paper begins with a brief section describing the data and methods used in the processing. The comparisons outlined in the preceding paragraph are described in the following section, and the paper concludes with a short section that discusses and summarizes the main results and outlines directions for future work.

\section{Data and Methods}

Sea level data are most commonly measured with tide gauges that consist of a float within a stilling well, which is generally a pipe approximately $30 \mathrm{~cm}$ in diameter. The well is closed to seawater with the exception of a vent hole that limits the rate at which water can enter the well, and its purpose is to mechanically filter sea level variability caused by high-frequency surface gravity waves. The fundamental measurement made by the tide gauge is simply the distance from the recording instrument to the float. Other types of gauges are also used, including gauges based on pressure transducers and gauges that measure the height of the water surface by observing the travel time of a reflected acoustic pulse. The float type gauge is the most common in the data set assembled for this study. In all cases the sea level data are referenced to a local set of benchmarks on the nearby land, as discussed in the previous section. More information on tide gauges is given by Pugh [1987].

The sea level data processing is straightforward and will only be described briefly. Additional details are available elsewhere [Kilonsky and Caldwell, 1991]. The sea level data are typically taken at a sampling rate ranging from several samples per second to several samples per hour. These high-frequency samples are averaged to obtain hourly data, which is the first "standard" sampling interval. These hourly data are used to compute daily sea level values that preserve sea level variability at timescales longer than about 2.5 days. The daily values are computed by subtracting estimates of the major diurnal and semidiurnal tides and then applying a convolution-type numerical filter. The long-period tides are not removed. At all stages of the processing, various quality control procedures are applied to the data.

Sea levels from tide gauges have in the past not been generally available until a year or more after collection. The World Ocean Circulation Experiment (WOCE) "Fast Delivery"'Sea Level Center recently established at the University of Hawaii [Mitchum, 1990] is intended to remedy this situation, particularly for the benefit of researchers wanting to access timely sea level data for use with the altimetry data. At the time of this writing, data from 71 sea level stations (Figure 1) have been assembled that cover at least part of the first 300 days of the TOPEX data set. These data are being made available to researchers worldwide. Sea level data have been contributed by groups and organizations in many countries and at present the only major source of data not yet represented are the ACCLAIM gauges [Spencer et al., 1993] from the South Atlantic and Southern Oceans. Thus the data set depicted in Figure 1 has no stations south of $50^{\circ} \mathrm{S}$.

As noted on the figure there are 43 island stations and 28 coastal ones. It is generally assumed that islands, at least small ones, should be more representative of open ocean conditions due to the lack of continental shelves and the associated boundary phenomena. I will not assume this from the start but will treat all gauges equally in the initial comparisons. I will, however, use separate symbols for island and coastal results in all figures. On the subject of island versus coastal gauges, it should also be noted that the altimeter measurements are probably not as reliable near the 


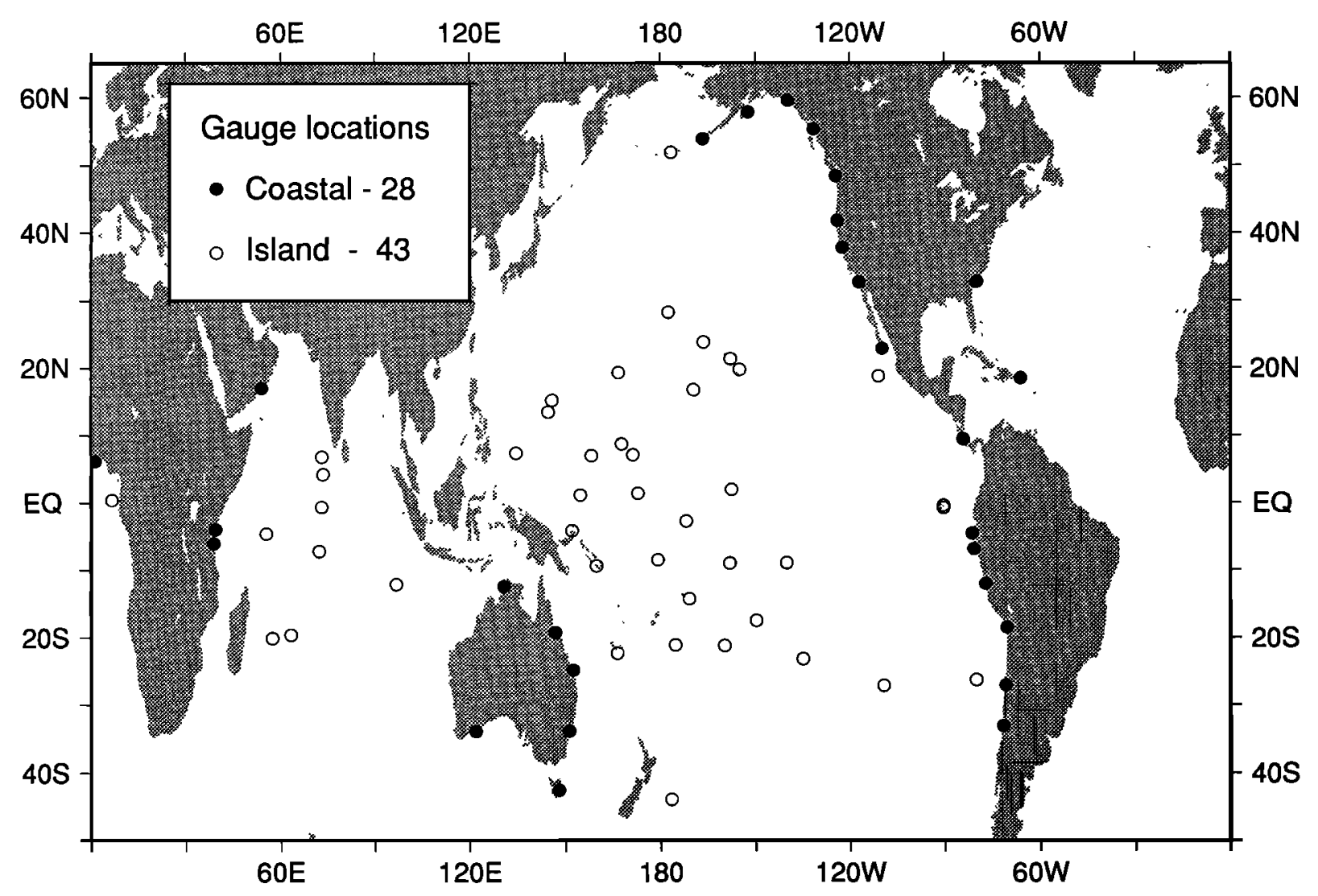

Figure 1. Locations of the stations providing sea level data for this study.

continental margins where most of the global sea level data are obtained. Consequently, the coastal measurements could be seen as providing a more stringent test of the altimeter's capabilities than the open ocean measurements, and good performance along the coastlines would be a convincing demonstration of the quality of the TOPEX sea surface height data.

In the present study only data from the TOPEX altimeter for the first 30 cycles are used. Significantly fewer passes of data have been obtained from the POSEIDON altimeter and comparison of those sea surface heights to the sea level data will be undertaken later. As indicated above, the processing of the TOPEX data to sea surface heights was done as recommended in the users handbook with only minor exceptions. For example, the computation of a standard pass geometry was accomplished by interpolating in latitude rather than longitude. Over most of the area covered by TOPEX this results in more accurate interpolations. Also, no inverted barometer correction was applied to the altimeter heights. This is necessary in order to be consistent with the sea level data, which are not subjected to an inverted barometer correction due to a lack of atmospheric pressure data at most stations. The most conservative set of data flags, as described in the handbook, were used except that data from shallow water depths was retained. In order to remove the geoid, data from cycle 18 was subtracted from all the cycles. Cycle 18 was chosen because it had relatively little missing data. Finally, as noted earlier, the Cartwright and Ray tide model was selected.

For each sea level station shown on Figure 1 data from the ascending and descending passes falling on either side of the tide gauge are separately used to form a comparison time series, resulting in up to four time series for each station. The sea surface height data are the 1/s data, which have an along-track spacing of approximately $5 \mathrm{~km}$. The sea surface height data were sampled at the latitudes corresponding to those of the tide gauges. The point thus chosen is within 2 $\mathrm{km}$ of the point of closest approach and is considered more conservative in that meridional sea level variations are larger than zonal ones over much of the ocean. The time of the altimeter passing is used to select the corresponding daily sea level value. No interpolation is done in either space or time.

In the initial comparisons each of these time series is analyzed separately; in the final comparison, after it is established that the separate passes are comparable, they are combined into a single time series for each station. Since cycle 18 data were subtracted from each pass in order to remove the geoid signal, the sea surface height time series are all zero at that cycle. The sea level time series, on the other hand, are referenced to a completely arbitrary level. The two types of time series were leveled together in the following fashion. First, the sea level daily time series for the period July 1992 through June 1993 were fit to a model consisting of a mean value and annual and semiannual harmonics. The addition of the harmonics prevent missing data from biasing the mean calculation. The fitted mean value is then removed from the sea level series. Second, a difference time series is computed by subtracting the sea surface height values from the corresponding sea level values. Then the sea surface height time series is corrected by the median value of the difference series. The median is 
Table 1. Percentage Points of the Distributions of the Correlations $(r)$ and RMS Differences $(\sigma)$ for the Initial Benchmark Case and Variations From It

\begin{tabular}{lcccccc}
\hline \multicolumn{1}{c}{ Case Description } & $r_{5}$ & $r_{50}$ & $r_{95}$ & $\sigma_{5}$ & $\sigma_{50}$ & $\sigma_{95}$ \\
\hline Initial benchmark case & -10 & 51 & 81 & 4.9 & 8.3 & 13.4 \\
Wet tropospheric correction & -23 & 31 & 71 & 6.6 & 9.7 & 14.9 \\
$\quad$ not used & & & & & & \\
25-km along-track smoothing & -11 & 54 & 81 & 4.5 & 7.9 & 13.6 \\
60-day harmonic removed & -12 & 57 & 87 & 4.1 & 6.7 & 12.0 \\
\hline
\end{tabular}

The correlations are in percent and the RMS differences are in centimeters. The subscripts define which percentage point is being tabulated; e.g., $5 \%$ of the correlation values lie below the values in the second column.

used as a robust estimator of the mean. The net effect of this leveling is to reference each pass of the sea surface height data to the mean sea level during July 1992 to July 1993, which is zero by construction.

The sea level and sea surface height time series computed in this way constitute what is referred to in the next section as the "initial benchmark case." These time series are considered to be similar to those that most users would arrive at independently. An alternate view is that these time series are a natural starting point for comparisons aimed at refining the series.

\section{Results}

\section{Analysis of the Initial Benchmark Case}

For each of the four passes at each station a correlation value and the RMS difference between the two time series are computed whenever more than 20 of the 30 cycles have valid data pairs. The results are summarized in Table 1, which gives the 5,50 , and $95 \%$ points of the distribution of the correlation and RMS values as computed over all the stations and passes. Characterizing the distributions by these percentiles rather than a mean and standard deviation minimizes the effect of outliers on the results. The $50 \%$ point, the median, is interpreted as the "typical" result. For the initial benchmark case the typical station thus has a correlation of 0.51 and an RMS difference of $8.3 \mathrm{~cm}$. Considering the islands and coastal stations separately yields values of $0.53 / 7.9 \mathrm{~cm}$ and $0.42 / 10.4 \mathrm{~cm}$, respectively. Given the simplicity of this initial comparison, these numbers are considered to be very good. There is no allowance made for any orbit error, for any tide model error, or for any other sort of error. There is also no smoothing in either space or time. To put these numbers in perspective, if the same calculation were performed on the Geosat sea surface heights from the exact repeat mission, the typical RMS difference would be of the order of the orbit errors, which range from tens of centimeters to meters depending on the orbit solution used.

The gross summary of the correlations and RMS differences provided in Table 1 does not give any information about potential patterns in the differences. The correlations and RMS values are plotted versus latitude in Figure 2. Plots were also done versus longitude, but no significant zonal patterns were detected. In Figure 2, however, it is apparent that the worst correlations are concentrated near $20^{\circ}-30^{\circ}$ latitude in both hemispheres and that these values are for island stations. Further, it is clear that the RMS differences are smallest in the tropics and are generally smaller for islands than for coastal stations. It is also interesting to note that the larger RMS values observed north of about $40^{\circ} \mathrm{N}$ are not associated with reduced correlation values.

The comparisons of the sea surface heights to the sea levels can also be used to evaluate the effectiveness of the various corrections applied to the sea surface heights during the processing. For example, Figure 3 shows the changes in the correlation and RMS values that occur when the wet tropospheric, or water vapor, correction is not used on the sea surface heights. Assuming that the water vapor correction used in the processing is working properly, then Figure 3 should show changes that are negative; that is, the comparisons degrade when these corrections are not done properly. The large decreases seen in both the correlations and RMS differences show that the water vapor correction is essential to the comparison. Also, from the median RMS values in Table 1 it is seen that not using this correction inflates the typical variance by almost $40 \%$. It is also clear from the plot versus longitude that the most extreme effect is in the tropics, which means that the changes are not associated with midlatitude, synoptic-scale weather systems. The fact that the changes due to neglecting the water vapor correction are so easily detected again indicates that orbit errors must be relatively small, as discussed in the introduction. Several other corrections were also checked as well. In all cases the results are the same as here, all of the corrections checked are improving the agreement between the sea surface heights and the sea levels.

In addition to evaluating the efficacy of the standard corrections applied to the sea surface heights, the sea levels can also be used to quantify the effect of additional processing steps that may be applied to either the TOPEX heights or the sea levels. Two cases of such additional processing are shown in Figure 4. In the left panel the changes due to smoothing the sea surface heights over approximately $25 \mathrm{~km}$ along the track are shown. While the changes are modest, they are consistently positive, indicating improvements in

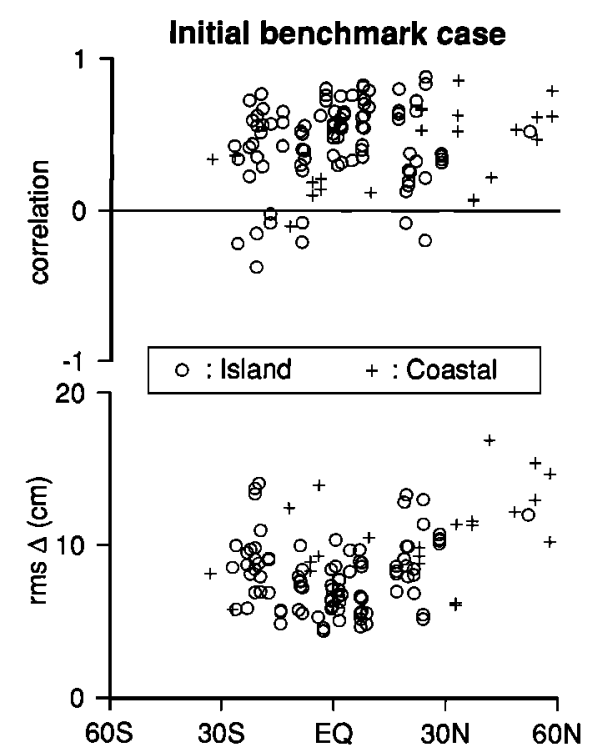

Figure 2. Correlations and RMS differences from the initial benchmark case (defined in the text) as a function of latitude. There are separate points for each of the four possible passes at each of the 71 stations. 


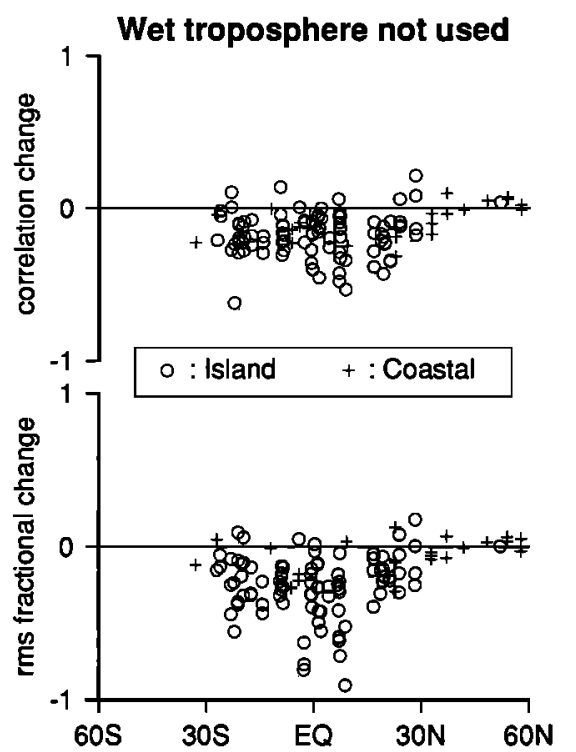

Figure 3. Changes in the correlation and RMS difference values relative to the initial benchmark case due to not applying the wet tropospheric correction. The correlation changes are positive if the modification to the processing results in a higher correlation than the initial benchmark case. The change in the RMS difference is normalized to the RMS difference value from the initial benchmark case and is positive if the RMS difference decreases with the modification to the processing. This choice for the sign convention means that beneficial changes result in positive values on both the correlation change and the RMS fractional change panels.

the comparison. This smoothing reduces the typical variance level by $9 \%$ from the initial benchmark case (Table 1). The $25-\mathrm{km}$ smoothing distance is not arbitrary but is chosen by evaluating the variance reduction obtained over a range of smoothing widths applied in space to the sea surface heights
Table 2. Variances, Averaged Over All Stations and Passes, Between Sea Surface Heights and Sea Levels as a Function of the Amount of Smoothing Applied

\begin{tabular}{ccccccc}
\hline & \multicolumn{5}{c}{ Sea Level Smoothing } \\
\cline { 2 - 7 } $\begin{array}{c}\text { Altimeter } \\
\text { Smoothing }\end{array}$ & 1 & 5 & 11 & 15 & 21 & 25 \\
\hline 1 & 77 & 75 & 76 & 76 & 77 & 75 \\
5 & 71 & 69 & 71 & 70 & 70 & 71 \\
11 & 72 & 69 & 70 & 69 & 71 & 72 \\
15 & 72 & 69 & 69 & 70 & 71 & 72 \\
21 & 73 & 69 & 70 & 70 & 71 & 72 \\
25 & 68 & 66 & 67 & 68 & 68 & 69 \\
\hline
\end{tabular}

Altimeter smoothing is applied along track and sea level smoothing is applied in time. Smoothing width is given as the number of points used in a median filter. Altimeter heights are spaced by approximately $5 \mathrm{~km}$ and sea levels are daily. Variances are in $\mathrm{cm}^{2}$. For example, the value in the second column and the second row, 71 $\mathrm{cm}^{2}$, results from smoothing the altimeter heights over $25 \mathrm{~km}$ and not smoothing the sea levels.

and in time to the sea levels (Table 2). Smoothing the sea surface heights over more than $25 \mathrm{~km}$ does not increase the variance reduction, and temporal smoothing of the sea levels does not help in any case. It is worth noting that the $25 \mathrm{~km}$ smoothing is consistent with early estimates of the alongtrack wavenumber spectrum for the sea surface heights from TOPEX that showed the wavenumber spectrum flattening into a white noise background at a length scale of about 25 $\mathrm{km}$.

The right panel of Figure 4 demonstrates a significant improvement in the comparisons due to the removal of a 60-day harmonic from the sea surface heights and sea levels. The choice of a 60 -day period is due to the fact that this is very close to the alias periods for both the $\mathbf{M}_{2}$ and $S_{2}$ tidal components. For a 300-day record length, neither component's alias period is distinguishable from a 60-day harmonic. In effect, removing this harmonic checks for errors due to inadequate tide removal from the sea surface heights.

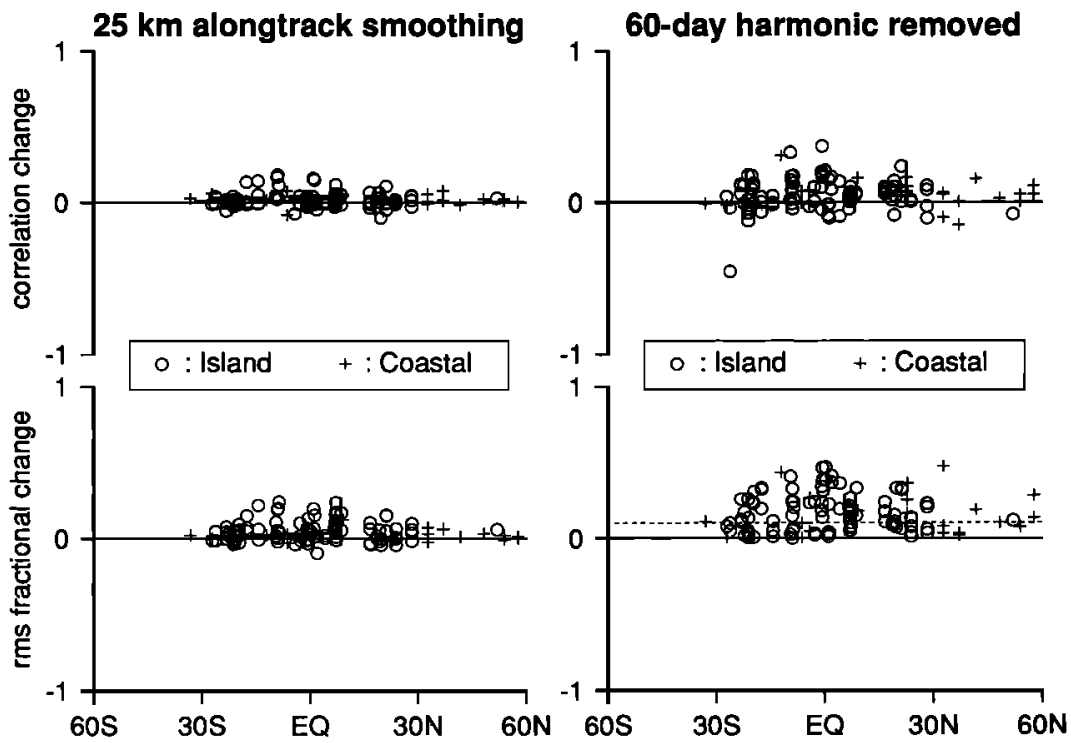

Figure 4. As in Figure 3, but for changes due to along-track smoothing of the sea surface height data (left panel), and due to the removal of a 60-day harmonic (right panel). Again, the changes are relative to the initial benchmark case. 


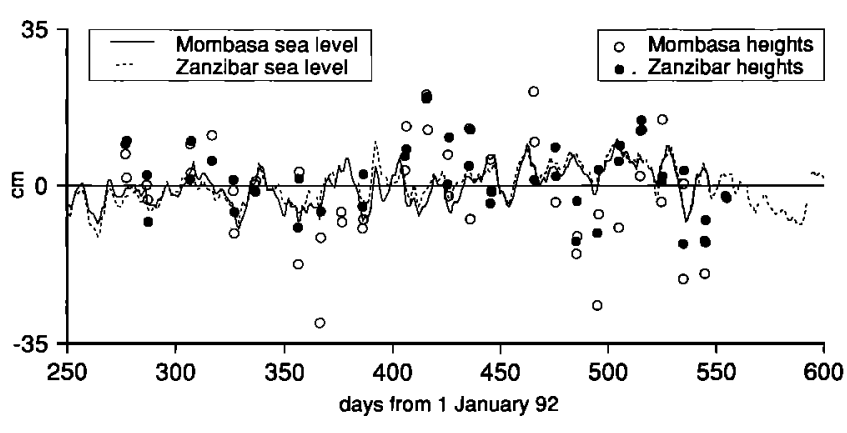

Figure 5. Time series comparison of sea levels at Mombasa (solid line) and Zanzibar (dashed line) along the eastern coast of Africa with the sea surface height values shown in open circles (Mombasa) and in solid circles (Zanzibar). The sea surface heights are from the modified benchmark case.

From Figure 4 and Table 1 it is seen that the typical variance of the difference between the sea surface heights and the sea levels decreases by $35 \%$. The correlations also improve slightly at most stations, though not as dramatically. Also, the improvements are largest in the tropics. There is, however, one station where the correlation decreases by almost 0.5. This station is San Felix, the island off the coast of South America near $30^{\circ} \mathrm{S}$ (Figure 1), and the reason for this decrease is unknown.

Of course, the fitting of a two-parameter model (the amplitude and phase of the 60-day harmonic) to the sea surface heights reduces the number of degrees of freedom in these short time series by approximately $10 \%$. Therefore one expects that even if the fit had no useful benefit that the variance would drop by order $10 \%$. The dotted line on Figure 4 's right bottom panel indicates this value and shows that the variance reduction at most stations is greater than this. Thus the conclusion is that correcting for residual $M_{2}$ and $S_{2}$ tidal signals significantly improves the comparison at most stations.

Similar harmonic fits were done at the alias periods corresponding to the $\mathrm{O}_{1}, \mathrm{~K}_{1}$, and $\mathrm{N}_{2}$ tides. None of these corrections produced the sort of uniform improvement shown in Figure 4. This is most likely due to the fact that these components are generally smaller in amplitude than $M_{2}$ and $S_{2}$, and consequently errors in the tide model are not as important for these components. This is not to say, however, that individual stations would not benefit. An example is found in the time series of sea level and sea surface height at Mombasa and Zanzibar along the coast of eastern Africa (Figure 5). My attention was drawn to Mombasa originally because the correlation and RMS values there were consistently poor. The initial suspicion was that the Mombasa sea level record had problems. But given the

Table 3. Percentage Points of the Distributions of the Correlations $(r)$ and RMS Differences $(\sigma)$ for the Modified Benchmark Case and Variations From It

\begin{tabular}{lrrrrrr}
\hline \multicolumn{1}{c}{ Case Description } & $r_{5}$ & $r_{50}$ & $r_{95}$ & $\sigma_{5}$ & $\sigma_{50}$ & $\sigma_{95}$ \\
\hline Modified benchmark case & -13 & 57 & 87 & 3.7 & 6.3 & 11.8 \\
1/rev correction applied & -14 & 59 & 85 & 3.5 & 5.8 & 12.3 \\
Propagation allowed & 2 & 51 & 84 & 4.0 & 6.5 & 13.7 \\
\hline
\end{tabular}

See Table 1 footnote for definitions. high correspondence between the sea levels at Mombasa and Zanzibar, which is located about $500 \mathrm{~km}$ to the south, this idea was rejected. From Figure 5 it can be seen that the differences between the sea surface heights and the sea levels have a periodicity comparable to the $K_{1}$ alias period of 173 days, indicating possible problems with that component of the tide model along the east coast of Africa.

\section{Analysis of the Modified Benchmark Case}

In the preceding section it has been established that a slight along-track smoothing of the sea surface heights results in improved comparisons. More significant improvements are obtained by removing a 60-day harmonic from the time series that corrects for tide model errors in the $\mathbf{M}_{2}$ and $S_{2}$ components. These two modifications were made to the data set and the result, which will be analyzed in more detail in this section, is referred to as the "modified benchmark case" in Table 3. The typical correlation value is now 0.57 and the RMS difference drops to $6.3 \mathrm{~cm}$, which is a $42 \%$ decrease in variance relative to the initial benchmark case. Note that to this point no sea level stations have been excluded from the analysis.

Again, it is useful to look at the meridional distribution of the correlation and RMS difference values (Figure 6). In comparison to the initial benchmark case (Figure 2), the correlations are generally higher, especially in the tropics where the correction with the 60-day harmonic had the most impact. Problems remain, however, at latitudes around $20^{\circ}-30^{\circ}$ in both hemispheres. It is also still apparent that the island stations have higher correlations and lower RMS difference values than the coastal stations. Specifically, the median values for island and coastal stations are $0.58 / 5.8 \mathrm{~cm}$ and $0.46 / 7.7 \mathrm{~cm}$, respectively. There is also less meridional variation in the values in Figure 6 as compared to Figure 2. The time series comparisons in Figure 7 are examples from the modified benchmark case. In this figure the comparisons at Baltra and Saipan illustrate the excellent results obtained in the tropics. Similar results are also seen in tropical

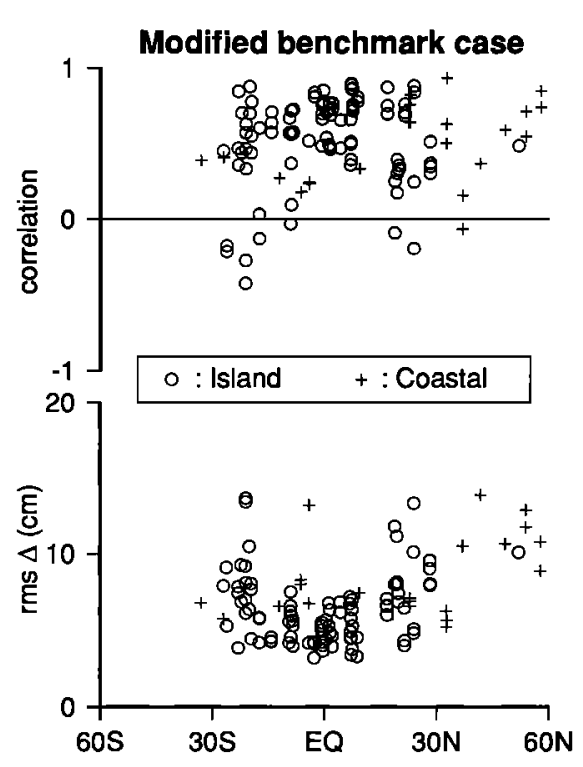

Figure 6. As in Figure 2, but for the modified benchmark case. The modifications defining this case are described in the text. 

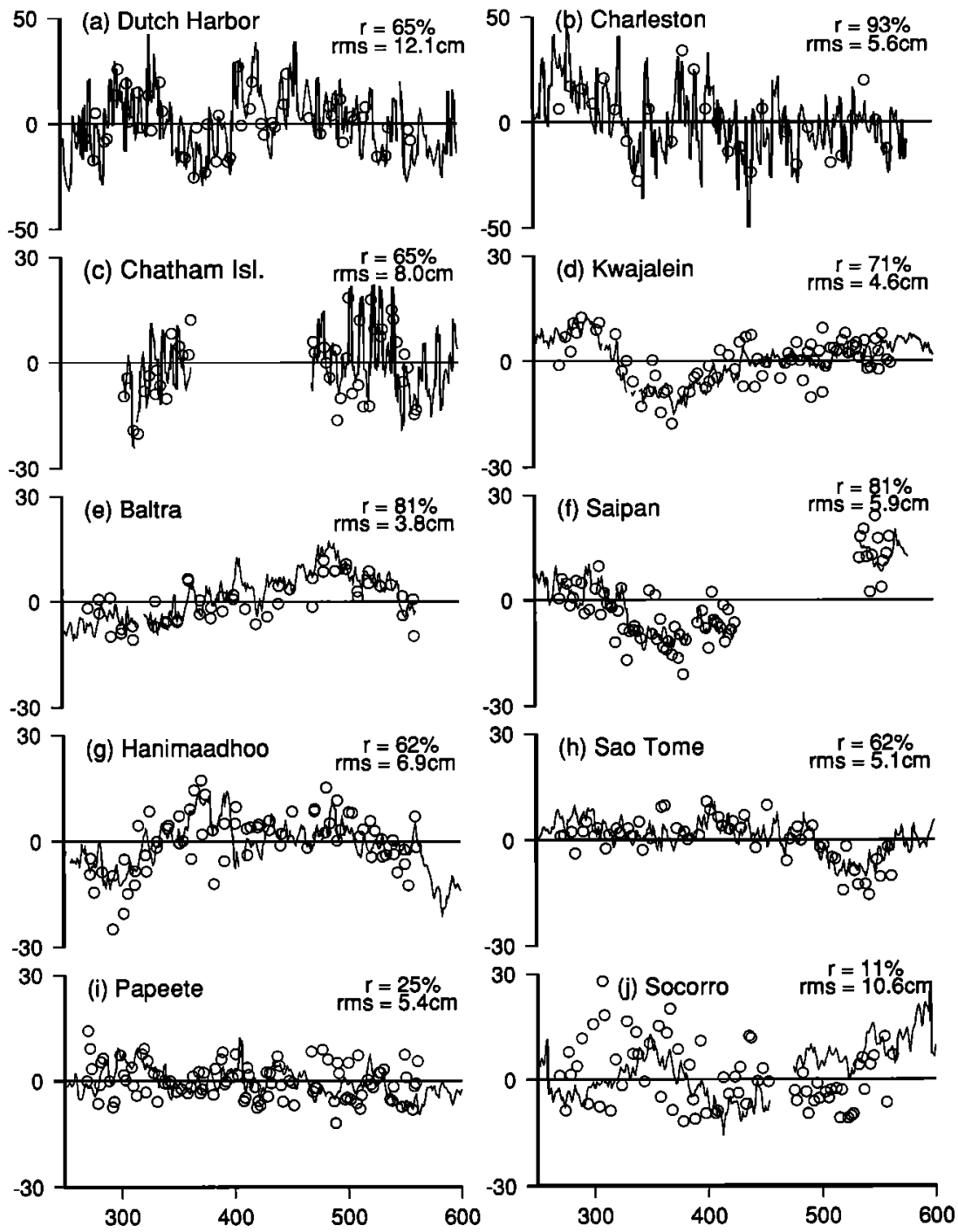

Figure 7. Time series from selected stations. The sea surface height values are taken from the modified benchmark case defined in the text. The sea level curves in each panel are the daily series referenced to the mean sea level at each station during the period July 1992 through June 1993. The sea surface height values are referenced to the same time period as described in the text. No distinction is made between the four altimeter passes, which means that up to four sea surface height values per 10-day cycle are possible. Note the change in the vertical axis scale on the top two panels.

stations outside the Pacific; for example, see Hanimaadhoo (Indian Ocean) and Sao Tome (Atlantic Ocean) in Figure 7.

As with the initial benchmark case, this new benchmark will be used to evaluate two further modifications to the processing. Specifically, these modifications involve empirical corrections for once per revolution orbit error and for propagating ocean signals. Again, the usefulness of these modifications will be evaluated by looking at the correlation and RMS difference changes relative to the modified benchmark case (Table 3 and Figure 8).

Most users of Geosat sea surface heights applied some sort of correction for once per revolution orbit errors. Often these corrections involved fitting the amplitude and phase of a once per revolution sinusoid. Corrections of this sort were suggested by Tai [1988] and have recently been extensively studied by Chelton and Schlax [1993]. In the present case a once per revolution sinusoid was fit that allows the amplitude and phase to vary fairly rapidly in time. This was accomplished by approximating the amplitude and phase of the once per revolution ( $1 / \mathrm{rev}$ ) harmonic by a sum of Chebyshev polynomials. The amplitude and phase functions were fit to 19 revolutions (less than 1 day) of the altimetric data at a time.

Applying this 1/rev correction (left panel of Figure 8) results in changes that are basically scattered about zero, meaning that this correction hurts at as many stations as it helps. A more subtle effect is also apparent in the plot of the changes in the correlations versus latitude. There appears to be a meridional trend to these changes, with decreased correlations in the northern hemisphere and change scattered more about zero near the equator and in the southern hemisphere. This is disturbing because it is the sort of pattern one would expect if the $1 /$ rev correction were simply suppressing the seasonal changes in the sea surface height data. This could happen because seasonal changes that are out of phase across the hemispheres would map on to a $1 /$ rev 


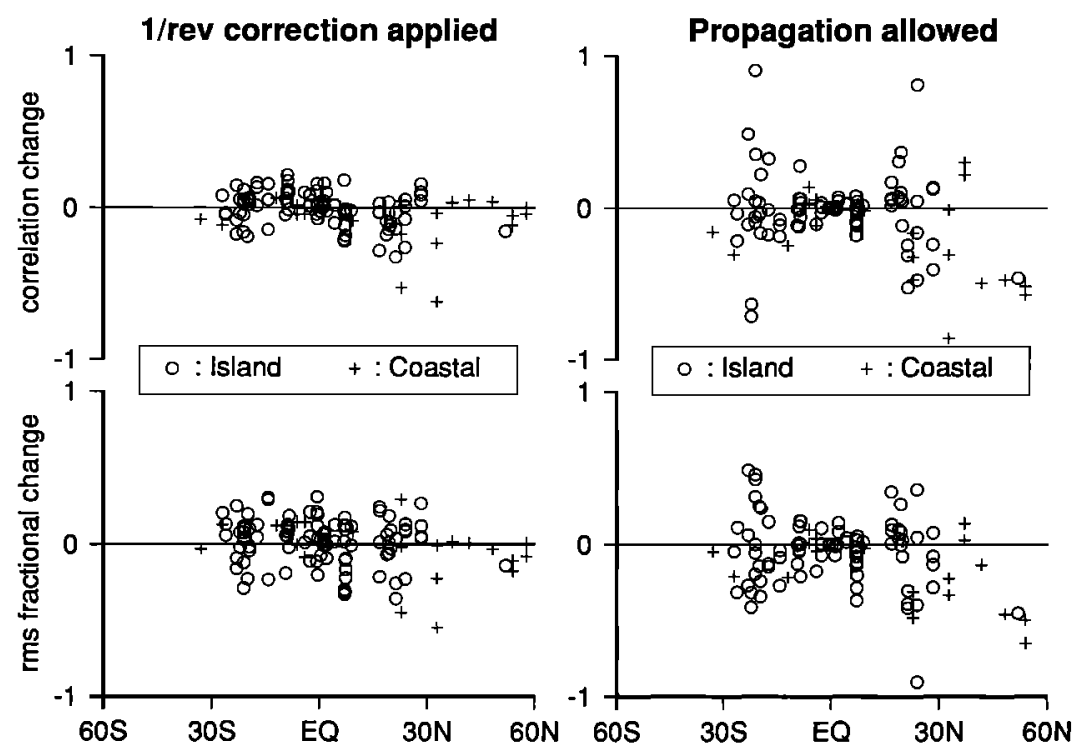

Figure 8. As in Figure 3, but for changes due to (left) 1/rev orbit error correction and due to (right) assuming that the signals are from propagating Rossby waves. Note that these changes are relative to the modified benchmark case plotted in Figure 6.

sinusoid. The fact that seasonal changes are larger in the northern than southern hemisphere would then account for a meridional trend in the correlation changes. In summary, the $1 /$ rev orbit error correction provides no clear improvement in the comparison of the sea surface heights to the sea levels. Further, the changes seen may be consistent with the $1 / \mathrm{rev}$ correction actually removing real oceanic seasonal signals from the data. No 1/rev corrections are used in the remaining analyses.

The right panel of Figure 8 shows the changes in the correlations and RMS differences that result from temporally shifting the pairs of sea surface height and sea level time series by an amount equal to the lag expected for first baroclinic Rossby waves. The amount of the lag is computed from the Rossby wave speed at the latitude of the station [Gill, 1982] and the zonal separation distance between the sea level station and the altimeter's ground track. For stations more than $5^{\circ}$ from the equator, the Rossby wave speed was estimated as $\beta c^{2} f^{2}$ with $c$ equal to $2.8 \mathrm{~m} / \mathrm{s}$. Within $5^{\circ}$ of the equator the speed was simply set to $0.9 \mathrm{~m} / \mathrm{s}$. One reason for checking for these propagation effects is that allowing for Rossby wave propagation has been shown to improve the correlation between Geosat sea surface heights and Wake Island sea levels from $4 \%$ to $65 \%$ (G. Mitchum, The source of 90-day oscillations at Wake Island, submitted to Journal of Geophysical Research, 1994). Also, van Woert and Price [1993] have demonstrated significant Rossby wave energy in Geosat sea surface heights north of the Hawaiian Ridge. Another reason is that the wider zonal spacing between TOPEX tracks, as opposed to Geosat, means that even larger temporal shifts are possible. For example, at $20^{\circ}$ latitude, Rossby waves propagate at about $6-7 \mathrm{~cm} / \mathrm{s}$, which implies a time lag of order 40-50 days between adjacent ground tracks.

An examination of the changes due to propagation in Figure 8 reveals both degradations and improvements in the correlations and RMS differences. The changes are small near the equator because wave speeds are fast here and the temporal lags are thus small. At midlatitudes to high latitudes most stations have poorer comparisons after this correction. On the other hand, there are some dramatic improvements, mostly at latitudes around $20^{\circ}-30^{\circ}$. Specifically, there are eight cases where the correlation increases by more than 0.4 and the RMS difference decreases by more than $10 \%$. Of these eight, seven are from stations within a few degrees of $20^{\circ} \mathrm{N}$ or $\mathrm{S}$. Of course, there are also stations at these latitudes whose comparisons degrade as well. This dilemma is partially resolved by noting that the seven stations that improve also tend to be found in the central and western portions of the basin. These results are very important considering the earlier discussion of the problems at these latitudes in Figures 2 and 7. One of the stations in this group is Rarotonga. To give a concrete example, the Rarotonga time series with and without the propagation correction are shown in Figure 9. The change is especially striking when one considers that the speed was not fit, but chosen a priori from theory.

But why are these latitudes special? To answer this, consider what must happen in order for the propagation correction to have any impact. First, the waves must be slow enough that significant time lags are introduced by the track to gauge spacing, which eliminates the low latitudes where propagation speeds are relatively high. Second, the Rossby wave portion of the total sea surface height signal must be dominant, or the statistics would not be sensitive to it. I speculate that this is not true at higher latitudes where wind and atmospheric pressure forcings are larger and where the time lags become so large that computing their exact magnitude is critical. Basically, then, the intermediate latitudes and longitudes away from the eastern boundary are especially sensitive to these waves because no other signals are available to drown them out, and because the wave speeds produce time lags large enough to scramble the signals if they are not corrected.

The propagation differences described above would be zero if every altimeter pass occurred directly over the sea 
level stations. Of course this is not what happens and it raises another issue. Namely, how much of the difference between the sea surface heights and the sea levels is due to the spatial separation between the two data sets? This question has been alluded to earlier in discussing the issue of how well tide gauge sea levels can represent the open ocean sea surface heights some distance away from the land. This question is addressed by considering the correlations and RMS differences as a function of how closely the altimeter approaches the tide gauge location (Figure 10). Some degradation of the comparisons with distance is indicated, especially for the correlations. Most of this tendency is removed, however, when the stations with strong propagating signals (solid squares) and low signal levels (solid circles) are removed from consideration. The propagating signals are not considered a fair test because the differences between the two data sets are not due to nonrepresentative tide gauges, but to real temporal lags. Similarly, stations with low signal levels (e.g., Papeete in Figure 3) would not improve if the approach were closer, because even a few centimeters of noise would still cause low correlations. Note that Wyrtki and Bongers [1987] have already reported that Papeete is located in an area of minimal sea level variability. In the case of the RMS differences, most of the larger values are associated with the propagating signals and the consequent large mismatch between sea level and sea surface height, or with stations that have large variability due to atmospheric pressure changes. These latter stations all have better than average correlations and the high RMS differences primarily reflect a much larger overall variability level rather than a poor match between the two data sets.

Once these effects are controlled for, the tendency for decreasing correlation or increasing RMS difference with distance is greatly weakened. But there is still a weak tendency that can be observed by concentrating on the upper
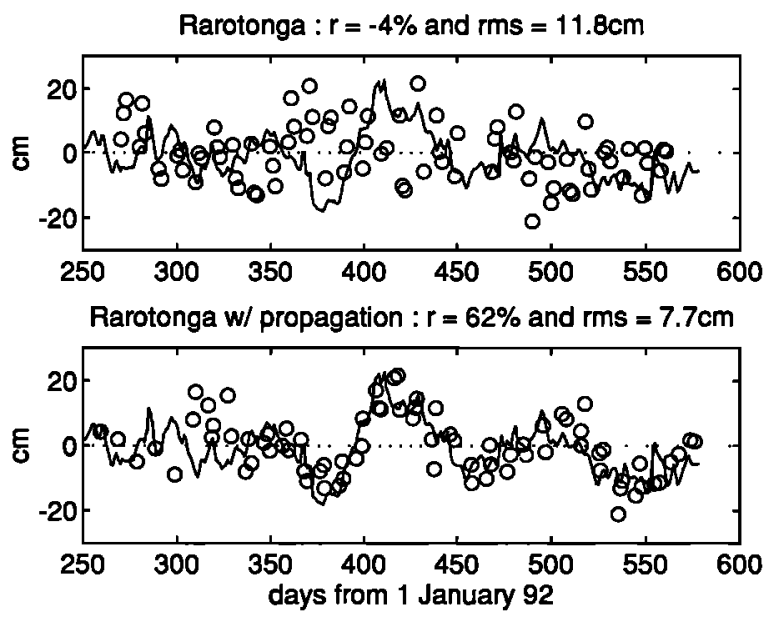

Figure 9. An example of a comparison that is improved by allowing for Rossby wave propagation. The solid curve is sea level at Rarotonga and is the same in the top and bottom panels. The open circles are the sea surface height values from the four passes nearest to Rarotonga. In the upper panel the values are plotted at the time of observation, while in the lower panel the times are offset by an amount equal to the lag derived from the Rossby wave speed (computed to be $5.9 \mathrm{~cm} / \mathrm{s}$ to the west) and the distance from the sea level station to the altimeter's ground track.

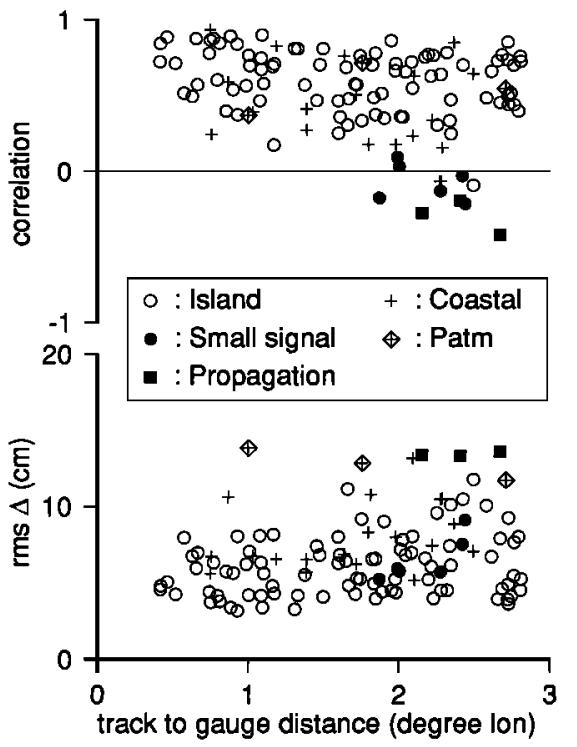

Figure 10. Correlations and RMS differences from the modified benchmark case plotted versus the distance from the altimeter ground track to the sea level station. These data are the same as those shown in Figure 6, with only the independent variable changed. The open circles and pluses are used as in all other plots. The solid circles mark comparisons with small correlations due to small sea level signals. The solid squares mark correlations that are small due to strong propagating signals. The diamonds with enclosed pluses show points with high RMS differences, but also high correlations, that are associated with large atmospheric pressure variability.

limit of the correlations versus distance in Figure 10. It is clear, however, that the decrease with distance is much smaller than the variation within any given range of distances.

To conclude this section I will make two final observations. First, there remain two stations with negative correlations in Figure 10, Fort Point on the northern California coast and the island of Socorro off the coast of Mexico. The Socorro comparison is shown in Figure 7. It is disappointing that both of the off equatorial islands off the coast of the Americas compare rather badly with the sea surface heights. It is suggestive that both of these records are from pressurerecording instruments. The differences are not due to atmospheric pressure, however. Second, separately considering only the coastal correlations versus gauge to track distance (see the pluses in Figure 10) does not reveal any strong trend either. This is encouraging because I expected that coastal signals would more likely be characterized by short spatial scales that should cause these stations to be less representative of open ocean conditions. This effect is not observed, however.

\section{Final Comparisons}

To this point the comparisons have all used correlations or RMS differences based on data from one altimeter pass or, at most, from the four passes closest to one tide gauge station. There is no reason, however, that the sea surface height and sea level data pairs from all the stations cannot be combined into a single set. As the altimeter revolves around the Earth the points of nearest approach (measured zonally) to the sea 


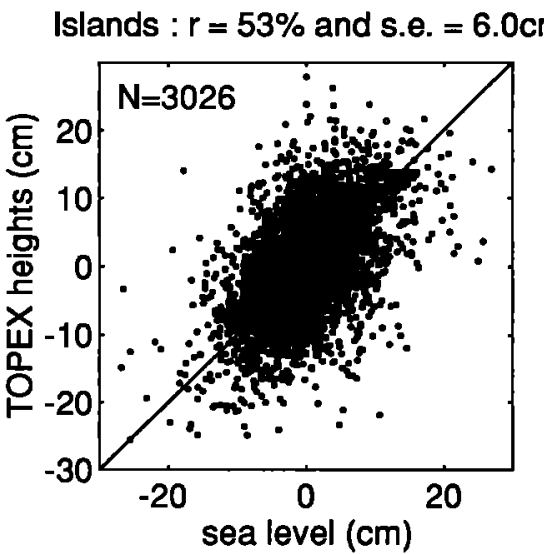

Coastal $: r=50 \%$ and $s . e .=8.0 \mathrm{~cm}$

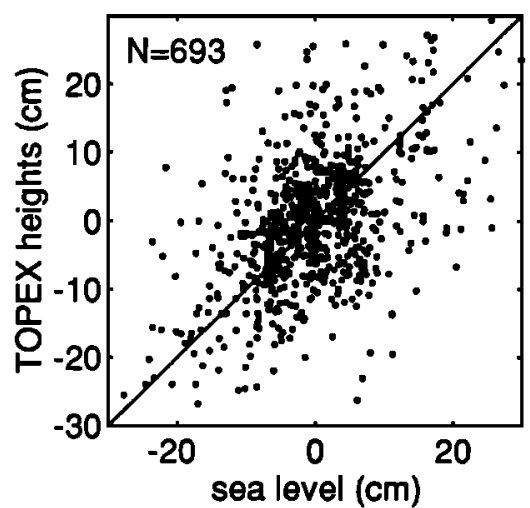

Figure 11. Scatterplot of all data pairs from the modified benchmark case. The top (bottom) panel includes data pairs from island (coastal) sea level stations. The scatter estimate (s.e.) is a robust estimator of the RMS difference between the data pairs. This estimate is computed as 1.4826 times the median of the absolute value of the differences after centering the differences on their median value. The factor 1.4826 forces this scatter estimate to be equal to the RMS value in the case of normally distributed differences.

level stations are identified. During each 10-day cycle, the time of these nearest approaches is used to pair the sea surface heights with the nearest daily sea level values. This procedure is allowed because the leveling scheme described in the data and methods section puts all of the data into a consistent reference frame; specifically, all of the heights and sea level are relative to the mean sea level computed over the time period July 1992 to July 1993 . The modified benchmark case, which includes 25 -km along-track smoothing of the sea surface heights and the removal of the 60-day harmonic, is used to generate such a set of data pairs.

A scatterplot of these data pairs is shown in Figure 11. Note that the data pairs are separated according to whether the sea level is from an island gauge or a coastal station. The larger differences at the coastal gauges noted earlier is very apparent here. To be precise, the variance using the coastal stations is 1.8 times that computed using the island gauges. But overall, the correlations are very satisfying. For example, the island correlation value of 0.53 is significantly different from zero at the $99.9 \%$ confidence level even if only 32 of the 3026 points are independent. The scatter estimate given on the figure (see the caption for a precise definition) is a robust estimate for the RMS difference. These values, $6 \mathrm{~cm}$ for the islands and $8 \mathrm{~cm}$ for the coastal stations, are also very encouraging.

An advantage to combining the data pairs in this fashion is that it allows a study of how the errors may change with time. Averaging the differences between the heights and sea levels within each 10-day cycle of the TOPEX data (Figure 12), for example, can be used as an independent check on the quality of the sea surface height data as the mission progresses. At present this figure indicates no long-term drift in the measurements, as measured by the average error over each cycle. Note that the leveling procedure used does not constrain these mean errors to lie near zero. If the satellite's computed orbital heights, for instance, were drifting, then this would show up on this figure. While there are no trends in the mean errors that is significant relative to the scatter estimates, there is an interesting, coherent variation over the last six cycles (25-30). This will be reexamined after more data are accumulated. Finally, I note that the scatter estimates are comparable from cycle to cycle, indicating that no portion of the mission is particularly noisy as compared to the rest.

From this point on I will concentrate on using the information collected thus far to derive estimates for how well the sea surface heights and sea levels agree after various problems are taken into account. To do this I will restrict the analysis to island stations because of the intrinsically higher RMS values that have consistently been observed at the coastal stations. I will also eliminate Socorro and San Felix, which have consistently been anomalous; that is, I assume that these stations have problems with the sea level measurements. I also eliminate the data pairs from Rarotonga and French Frigate Shoals because of the large changes due to the Rossby wave propagation discussed above. Finally, I will low-pass filter the data by computing the median values of the sea surface heights and the sea levels from each of the four passes that occur during each cycle, requiring that at least three passes be available to accept the low-passed data. This slight smoothing eliminates day to day variability from both data sets in order to better estimate the errors on longer timescales. An example of a smoothed comparison is shown in Figure 13. The comparison is excellent, despite the fact that the smoothing applied to the solid curve was a simple 10-day running mean rather than the median filtering used on the sea surface heights. But note carefully that this station is not chosen to be typical but to demonstrate how good the smoothed comparisons can be.

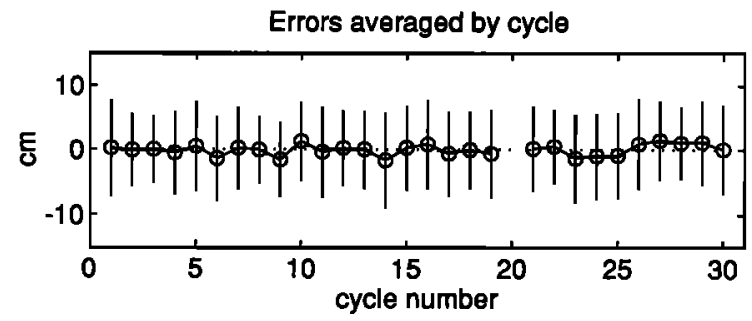

Figure 12. Time series of sea surface height and sea level differences averaged by cycle. The open circles mark the mean values, and the error bars are \pm 1 standard deviation. The standard deviations are from the scatter estimate defined in the caption of Figure 11. Cycle 20 had no data from TOPEX. Cycle 1 begins on day 266 in the reference system used in the other time series plots. 
Selecting data pairs according to the above principles and applying the 10-day smoothing produces 552 data pairs. The scatterplot (Figure 14) reveals an excellent correspondence between the sea surface heights and the sea levels, with a correlation of 0.66 and a scatter estimate (i.e., RMS difference) of $4.3 \mathrm{~cm}$. This is my best present estimate of the basic level of agreement between these data sets. Since only island stations are used, the analysis is unfortunately biased to the tropics (Figure 1), and the tropical Pacific in particular. As more sea level data become available in the future, this can to some extent be ameliorated. Finally, note that the 10-day smoothing reduced the RMS difference at the islands stations from $6.0 \mathrm{~cm}$ to $4.3 \mathrm{~cm}$. If a similar improvement were to be obtained at the coastal stations, then the expected RMS difference along the coast for timescales longer than 10 days would drop to order $6 \mathrm{~cm}$, which is significantly smaller than many of the interesting signals found along continental margins.

\section{Discussion}

The first analyses used what was referred to as the initial benchmark case, which consists of the sea surface heights after applying only the standard processing as defined in the data handbook. Correlations and RMS differences were computed separately for the time series resulting from each of the four nearest altimeter passes at each station, and typical values were estimated from the median values. This analysis results in a median correlation of 0.53 for the island tide gauges and $\mathbf{0 . 4 2}$ for the coastal stations. The median RMS differences were $7.9 \mathrm{~cm}$ for the islands and $10.4 \mathrm{~cm}$ for the coastal gauges. While these numbers are considered to be very good for such a simple intercomparison, it is more noteworthy that the RMS differences have a lower bound of order $5 \mathrm{~cm}$ (Figure 2), which is comparable to the 5\% point of the distribution of the RMS differences (Table 1). This sets an upper bound for errors in the sea surface heights (or the sea levels) that do not have any strong geographical dependence; that is, if there are errors with a $5 \mathrm{~cm}$ RMS that do not have any preferred spatial dependence, then the RMS differences with the ground truth data can not go much below this level. Assuming that the orbit errors are of this type, then one concludes that the orbit calculations are good to better than $5 \mathrm{~cm}$, which is significantly better than the design criteria for the mission. And given that orbit errors are not

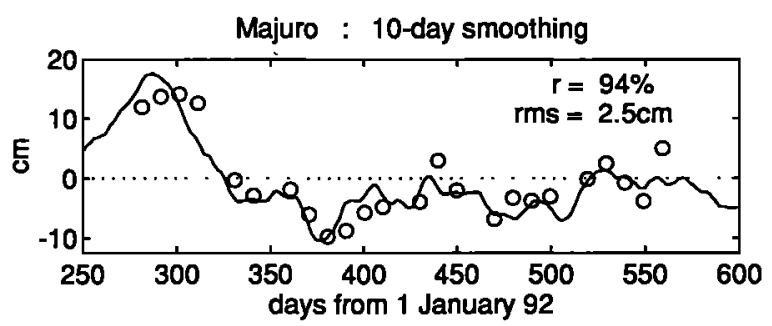

Figure 13. Time series comparison at Majuro after 10-day smoothing. The sea level (solid curve) is the output of a 10 -day running mean filter applied to the daily values. The sea surface height data (open circles) are the median values of the four passes from each cycle of the TOPEX data. The median value is computed for each cycle where at least three passes return a sea surface height data point.

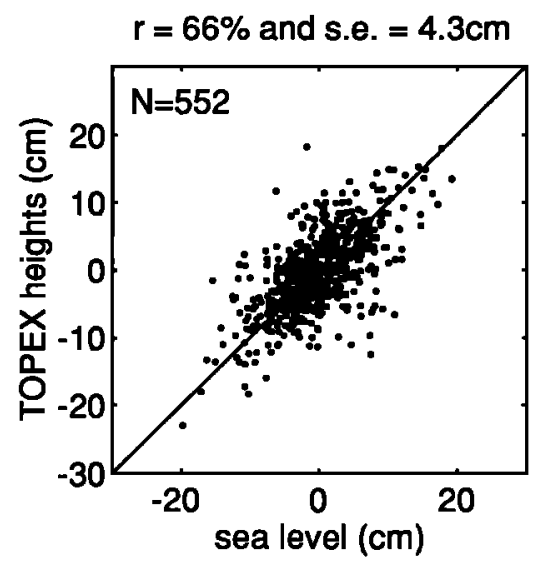

Figure 14. Scatterplot of the sea surface height and sea level data pairs selected from the modified benchmark case. Smoothing over 10-day intervals is performed before the data pairs are selected. The details of the selection are described in the text, but it is primarily a selection of island, as opposed to coastal, stations. The scatter estimate is a robust estimate of the RMS difference between the data pairs (see caption of Figure 11 for definition).

the only contributor to this noise floor, it is probable that the orbit calculations are even better.

Additional analyses of the initial benchmark case shows that two additional processing steps greatly improve the comparison between the sea surface heights and the sea levels. First, the sea surface height data are smoothed along track for a distance of about $25 \mathrm{~km}$. This length scale is chosen empirically in this study, but it is consistent with previous estimates of the length scale where along-track wavenumber spectra flatten into a white noise background. Second, and more importantly, removing a 60-day harmonic from the heights and sea levels before the comparison typically reduces the variance of the differences by $35 \%$. This is the most effective adjustment to the data identified in this study. The reason for removing a 60-day harmonic is that it captures the aliased energy of mismodeled $\mathbf{M}_{2}$ and $\mathbf{S}_{2}$ tidal variations. The conclusion is that tide model errors are the largest single source of discrepancies between the two data sets. From the pattern of changes (Figure 4) it appears that this correction is most effective in the tropics. Applying these additional processing steps to the sea surface heights and sea level data sets results in median correlations of 0.58 for the islands and $\mathbf{0 . 4 6}$ for the coastal stations. The corresponding RMS differences are $5.8 \mathrm{~cm}$ and $7.7 \mathrm{~cm}$. The lower bound for the RMS values, which is again interpreted as an upper bound for the orbit errors, is now significantly lower at a value of about $3 \mathrm{~cm}$.

Further analysis of the modified benchmark produces several additional results. First, there are a number of stations, particularly at latitudes around $20^{\circ}-30^{\circ}$ in both hemispheres, where allowing for signal propagation at the local Rossby wave speed results in significant, and sometimes dramatic, improvement. At Rarotonga $\left(21^{\circ} \mathrm{S}, 160^{\circ} \mathrm{W}\right)$ the changes are maximum, with the correlation increasing from -0.04 to 0.62 and the RMS difference decreasing from 11.8 to $7.7 \mathrm{~cm}$. Second, although removing harmonics due to tidal components other than $M_{2}$ and $S_{2}$ did not result in overall improvement, there were specific comparisons at 
stations along the eastern coast of Africa that indicated problems with the $\mathrm{K}_{1}$ tide there. Third, and perhaps most significant for researchers familiar with Geosat data processing, attempting to correct for a once per revolution orbit error does not result in any useful improvement in these comparisons. In fact, there is an indication that making this sort of adjustment actually degrades the comparisons by suppressing the seasonal variability in the sea surface height data.

Before attempting to make final estimates of the errors in the sea surface height data, the time series for each station were slightly smoothed in time to eliminate noise at timescales less than the altimeter cycle length (10 days). This smoothing occurs after the $25-\mathrm{km}$ along-track smoothing and the removal of the 60-day harmonics that define the transition of the data from the initial to the modified benchmark case. Also, only data pairs derived from island sea level stations were retained. The remaining 552 data pairs have a correlation coefficient of 0.66 ; the RMS difference between sea surface height and sea level is $4.3 \mathrm{~cm}$. From this comparison I conclude that the RMS total error of the altimeter is less than about $4 \mathrm{~cm}$ for timescales longer than 10 days. If the error in the sea level measurements is taken into account, this upper bound for the RMS errors in the altimetric heights will probably drop to several centimeters. It is not appropriate to compare this RMS difference of several centimeters to the 4-5 cm RMS differences between Geosat heights and sea levels that were cited in the introduction. The Geosat values apply on timescales greater than a few months, whereas the TOPEX values are obtained after smoothing over only 10 days. If the errors are white in frequency space, this implies that the TOPEX variance is approximately an order of magnitude smaller than that of Geosat.

The assessment in the preceding paragraph is biased toward tropical islands, and tropical Pacific islands in the particular. The correlations observed at the coastal stations were also very reasonable (Figure 6 and Table 3), but the RMS differences were generally about 33\% larger. But given the higher amplitudes and the shorter timescales of the variability along the coastlines, it is surprising that the altimeter does so well. An example of an excellent coastal comparison is found at Charleston (Figure 7b) on the east coast of North America. Despite large amplitude fluctuations on timescales of days to weeks, the sea surface heights match the sea levels well enough to produce a correlation of 0.94 , with a RMS difference of less than $6 \mathrm{~cm}$. This station compares better than other coastal stations from the west coast of North America, but I note that the continental shelf along the east coast is much wider, of the order of 100-200 $\mathrm{km}$ across. One explanation for the high correlation at Charleston could be that the altimeter is providing high enough quality data on the shelf to capture the coastally trapped signals that account for much of the coastal variance.

Analyses such as those described in this paper will be continued throughout the TOPEX/POSEIDON mission. These comparisons act as a continuous check on the "health" of the complex system that provides the final sea surface heights and will increase confidence in the altimetric data. In addition, the sea surface heights will provide checks on the sea level data being assembled for the WOCE project. An excellent example of this application is seen in the time series at Saipan (Figure 7f). Note the large change in the level across the data gap extending from approximately day 430 to day 530. Normally, such a sea level record would be considered suspect because experience shows that a large level change after a period of data loss is a common indicator of a problem with the station's datum, or reference height. The addition of the sea surface height data from TOPEX, however, confirms that the level change is real. The net result is that the comparison of the sea surface heights and the sea levels results in increased confidence in both data sets.

Acknowledgments. Data management and programming assistance was provided by Shikiko Nakahara, Bernard Kilonsky, and David Stone. They performed extremely well under pressure and this paper would not have been possible without their help. I benefitted from many helpful discussions with my colleagues, especially Fred Bingham, Steve Chiswell, Eric Firing, Dennis Moore, Fritz Schott, and Klaus Wyrtki. Sea level data were obtained from the University of Hawaii Sea Level Center and the National Ocean Service in the United States, from the National Tidal Facility at Flinders University in Australia, and from ORSTOM in France. This work was supported by NOAA through the Joint Institute for Marine and Atmospheric Research Cooperative Agreement NA37RJ0199 and by NASA through the Jet Propulsion Laboratory as part of the TOPEX Altimeter Research in Ocean Circulation Mission. This is contribution 3630 of the School of Ocean and Earth Science and Technology, University of Hawaii; JIMAR contribution 94-0276.

\section{References}

Cartwright, D., and R. Ray, Oceanic tides from Geosat altimetry, $J$. Geophys. Res., 95, 3069-3090, 1990.

Chao, Y., D. Halpern, and C. Perigaud, Sea surface height variability during 1986-1988 in the tropical Pacific Ocean, J. Geophys. Res., 98, 6947-6960, 1993.

Chelton, D., and M. Schlax, Spectral characteristics of timedependent orbit errors in altimeter height measurements, $J$. Geophys. Res., 98, 12,579-12,600, 1993.

Cheney, R., B. Douglas, and L. Miller, Evaluation of Geosat altimeter data with application to tropical Pacific sea level variability, J. Geophys. Res., 94, 4737-4748, 1989.

Gill, A., Atmosphere-Ocean Dynamics, Academic, San Diego, Calif., 1982.

Harangozo, S., P. Woodworth, R. Rapp, and Y. Wang, A comparison of monthly mean sea level variability determined from Geosat altimetry and a global tide gauge dataset, Int. J. Remote Sens., 14, 789-795, 1993.

Kilonsky, B., and P. Caldwell, In the pursuit of high-quality sea level data, IEEE Oceans Proceedings, 2, 669-675, 1991.

Koblinsky, C., P. Gaspar, and G. Lagerloef (Eds.), The Future of Spaceborne Altimetry: Oceans and Climate Change, 75 pp., Joint Oceanographic Institutions, Washington, D. C., 1992.

Mitchum, G., U.S. WOCE supports global sea level data collection, WOCE Notes, 2(6), pp. 10-12, Tex. A\&M Univ., College Station, Tex., 1990.

Mitchum, G., and B. Kilonsky, Observations of tropical sea level variability from altimeters, in Oceanographic Application of Remote Sensing, edited by M. Ideda and F. Dobson, CRC Press, Boca Raton, Fla., in press, 1994.

Pugh, D., Tides, Surges and Mean Sea-Level, John Wiley, New York, 1987.

Spencer, R., P. Foden, C. McGarry, A. Harrison, J. Vassie, T. Baker, M. Smithson, S. Harangozo, and P. Woodworth, The ACCLAIM programme in the South Atlantic and Southern Oceans, Intl. Hydrogr. Rev., 70, 7-21, 1993.

Tai, C.-K., Geosat crossover analysis in the tropical Pacific, 1, Constrained sinusoidal crossover adjustment, J. Geophys. Res., $93,10,621-10,629,1988$.

Tai, C.-K., W. White, and S. Pazan, Geosat crossover analysis in the tropical Pacific, 2, Verification analysis of altimetric sea level 
maps with expendable bathythermograph and island sea level data, J. Geophys. Res., 94, 897-908, 1989.

van Woert, M., and J. Price, Geosat and advanced very high resolution radiometer observations of oceanic planetary waves adjacent to the Hawaiian Islands, J. Geophys. Res., 98, 14,619$14,632,1993$.

Wyrtki, K., and T. Bongers, Sea level at Tahiti-A minimum of variability, J. Phys. Oceanogr., 17, 164-168, 1987.

Wyrtki, K., and G. Mitchum, Interannual differences of Geosat altimeter heights and sea level: The importance of a datum, $J$. Geophys. Res., 95, 2969-2975, 1990.

G. T. Mitchum, Department of Oceanography, University of Hawaii at Manoa, MSB 307, Honolulu, HI 96822-2336.

(Received November 3, 1993; revised June 21, 1994; accepted June 21, 1994.) 